\title{
Laser Cooling System for Magnetic Resonance Imaging Scanner using Normal Controller
}

\author{
Roshini T. V. \\ Research Scholar \\ Department of ECE \\ Karpagam Academy of \\ Higher Education, Coimbatore
}

\author{
Kamalraj Subramaniam \\ Associate Professor \\ Department of ECE \\ Karpagam Academy of \\ Higher Education, Coimbatore
}

\begin{abstract}
Magnetic Resonance Imaging (MRI) is a diagnostic procedure that uses a combination of large magnet, radio frequencies and computer to produce detailed image of organs and structures within the body. The MRI scanner has a large magnet with a massively strong magnetic attraction, which is responsible for scanning. The magnet in the MRI scanner creates much larger electric current, creating intense magnetic fields which cause high heat in the scanner and this can bring in undesirable effects. This paper investigates the problem of heat in MRI scanner which may lead to inaccuracy to results and hazards to patients. To ensure this issue, various existing methods were employed but this work will act as an effective and flexible remedy. This system is instigated in MATLAB/Simulink platform. A cooling system for MRI scanner based on LASER Technology is presented. Laser Cooling System (LCS) is a resonant technology used for cooling an object to an absolutely quite low temperature by around $40 \mathrm{~K}$. The presented cooling system for MRI scanner using laser provides optimal cooling, the system has less cost and the complexity associated with handling is also less.
\end{abstract}

\section{Keywords}

Magnetic resonance imaging, super conducting magnet, laser cooling system, semiconductor ring laser

\section{INTRODUCTION}

MRI is an influential symptomatic instrument by which medicinal group uses for visualization of delicate tissues [1], [2]. Since production of the first human self-perceptions in 1977, MRI has turned into one of the essential devices in therapeutic diagnostics. The low-field whole-body MRI magnets $(<0.35$ tesla) are a mix of resistive magnets with iron yoke and permanent magnets. Resistive magnets have the lowest installation cost among all types of MRI systems but require a large power consumption. The permanent-magnet MRI systems are heavy. Their installation cost is rather high but maintenance cost is low. The low-field magnets typically have relatively poor uniformity and stability. Poor uniformity results in poor image quality, although it might be adequate for some applications [3]. The magnetic field required for this is done by superconductivity or reducing the resistance in the wires to almost zero [4]. To do this, the wires are persistently washed in fluid helium at 452.4 degrees underneath zero

Fahrenheit $\left(-269.1^{\circ} \mathrm{C}\right)$ [5]. A typical MRI scanner uses 1,700 liters of liquid helium, which needs to be topped up

periodically, around 30 liters of liquid helium has to be added for every two and a half months. The helium fluid is highly expensive and the treatment of the helium fluid is troublous, because it is effortlessly exposable [6]. In this sense, our work is focused on creating a high effective LCS for MRI scanner
LASER is a device that releases light by optical intensification utilizing the stipulated emission of electromagnetic radiation [7], [8]. Since 1975, LASER radiation has been utilized with the end goal of cooling the gases [9]. LCS works on the fundamental guidelines of Doppler Effect [10].

\section{MRI}

MRI is a medical imaging technique used in radiology to visualize the anatomy and physiological process of the human body. Since MRI is operated at Radio-Frequency range, unlike the other imaging modalities such as Computer Tomography (CT), Single Photon Emission Computed Tomography (SPECT), and Position Emission Tomography (PET), there is no ionizing radiation [11]. But it has a strong magnetic field [12].

MRI systems use a superconducting magnet, which consists of many coils or windings of wire through which a current of electricity is passed, creating a magnetic field of up to 2.0 tesla [13]. While superconductive magnets are expensive, the strong magnetic field allows for the highest quality imaging. Fig. 1 shows the schematic of MRI scanner [14]

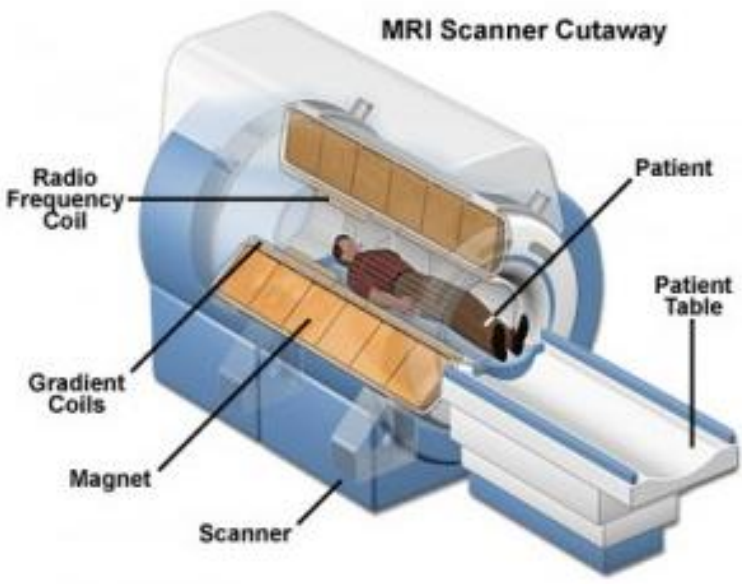

Fig. 1 Schematic of MRI scanner

The magnet of an MRI scanner provides a stable, strong and spatially uniform magnetic field within a structure that allows adequate patient access.

\subsection{Working of MRI}

The human body is largely made of water molecules, which consists of smaller hydrogen and oxygen atoms. Proton lies at the center of each atom, which is sensitive to any magnetic fields and hence this proton serves as a magnet. Normally the water molecules in our body are randomly arranged, but upon 
entering on the MRI scanner the first magnet causes the body's water molecules to align in one direction. The second magnet was then turned on and off in a series of quick pulses, causing each hydrogen atom to alter their alignment and quickly switches back to their original relaxed state, when switched off. Although the patient cannot feel these changes, the scanner can detect them and in conjunction with a computer can create a detailed cross sectional image for the radiologist.

\section{COOLING SYSTEM IN MRI SCANNER}

The primary component of MRI is the magnet that maybe a superconductive, permanent or resistive. But superconductive magnet is the most commonly used since it has high performance factor than others. While performing MRI generates enormous amount of heat due to the presence of superconductive magnet [14]. Superconductive magnet has marvelous electric field due to this only the heat is generated. The heat in MRI may affect its performance. So we have to control it. For that we use cooling system.

\subsection{Helium Cooling System}

Helium Cooling System can be favorable when it is very difficult to directly connect a cooler to an application due to whatever restriction there may be safety, space, noise, vibration, rotating devices etc. The advantage of gaseous Helium is its operation window. It can be used at any temperature between ambient down to approximately 10 Kelvin. Restrictions that you might have with other fluids like liquefaction and or freezing are no issue with Helium gas. Sufficient density is available for proper heat transfer, If the gas is pressurized (preferably in a range of 10-20).

During the working of MRI machine a very large magnetic field using a super conducting magnet was generated, a current was passed through many coils. A lot of energy is required for generating large magnetic field and is accomplished by super conductivity and resistance of the wire should reduce almost zero. This is done by bathing the wires in a continuous supply of liquid helium at -249 Kelvin [6]. A typical MRI scanner uses 1,700 liters of liquid helium, which needs to be topped up periodically. The majority of the world's helium supply is created through natural radioactive decay and cannot be artificially synthesized, as the gas is a non-renewable resource. To overcome the difficulties of liquid Helium, LCS is employed.

\subsection{LCS}

LCS is one of the recent technologies used to cool magnet in MRI. The temperature of a laser system can determine its lifetime, performance and safety. Many systems simply burn out if they are too hot for too long. Thermal lensing damages the beam shape of most solid-state lasers, and the output wavelength of laser diodes closely depends on their temperature.

In laser cooling, atomic and molecular samples are cooled down to nearly absolute zero through the interaction with one or more laser fields. The basic principle of laser cooling is Doppler effect [15]. In Doppler effect the frequency of light is tuned slightly below an electronic transition in the atom. Because the light is detuned to lower frequency, the atom will absorb more photons if they move towards the light source. If light is applied from two opposite directions, the atom will scatter more photons. If this process continuous, the speed of the atom reduces and hence the kinetic energy also reduces. Which reduces the temperature of the atom, and hence cooling of the atom is achieved [16].

As per Doppler cooling, if a stationary atom sees the laser neither red-shifted nor blue shifted, it does not absorb the photon. An atom moving away from the laser sees that the laser is red shifted, then also it does not absorb photon. If an atom is moving towards the laser and sees that it is blueshifted, the it absorbs the photon and thus the speed of the atom will get reduced. The absorbed photon excites the atom, and the electron will move to the higher energy state and the atom reemits the photon. As the direction of the emission is random, there is no net change in momentum over many absorption-emission cycles [17].

\section{LCS FOR MRI SCANNER}

LCS is a recent technology used cooling an object. The principle behind this is Doppler effects; it reduces the motion of an atom or molecule by the application of a force in opposite direction. We model the LCS with a normal controller for the cooling of MRI scanner. The proposed systems are implemented in the working platform Matlab/Simulink. The performance of the model is analyzed.

LCS can maintain the superconducting magnet below critical temperature. So our aim is to model an optimal LCS which can maintain 10 Kelvin cooling. From the previous research work related to LCS, it is found that the temperature reduction is completely depends on the wavelength of the laser beam. In our paper we are target to model the system so that the system can provide cooling up to 10 Kelvin.

In the proposed method we develop an LCS which can maintain the super conducting magnet below its critical temperature. So our aim is to model an optimal LCS which can maintain 10 Kelvin of cooling. From the previous research work related to LCS, we have found that the temperature reduction is completely depend on the wavelength of the laser beam. In [6], Jun Zhanget al has obtained 10 Kelvin for semiconductor device cooling at the wavelength of $514 \mathrm{~nm}$ and at $532 \mathrm{~nm}$ they got 15 Kelvin of cooling. So in our paper we are target to model the system so that the system can provide cooling up to 10 Kelvin. The block diagram of proposed system is shown in Fig. 2.

Here we concentrate more on cooling system in MRI scanner. The main source of heat generation is super conducting magnet. A super conducting magnet is an electromagnet made from coils of superconducting wire. In its super conducting state the wire can conduct much larger electric current than ordinary wires, creating intense magnetic fields. Super conducting magnets can produce greater magnetic fields than all but the strongest electromagnet can be cheaper to operate because no energy is dissipated as heat in the windings. They must be cooled to cryogenic temperature during the operation. 


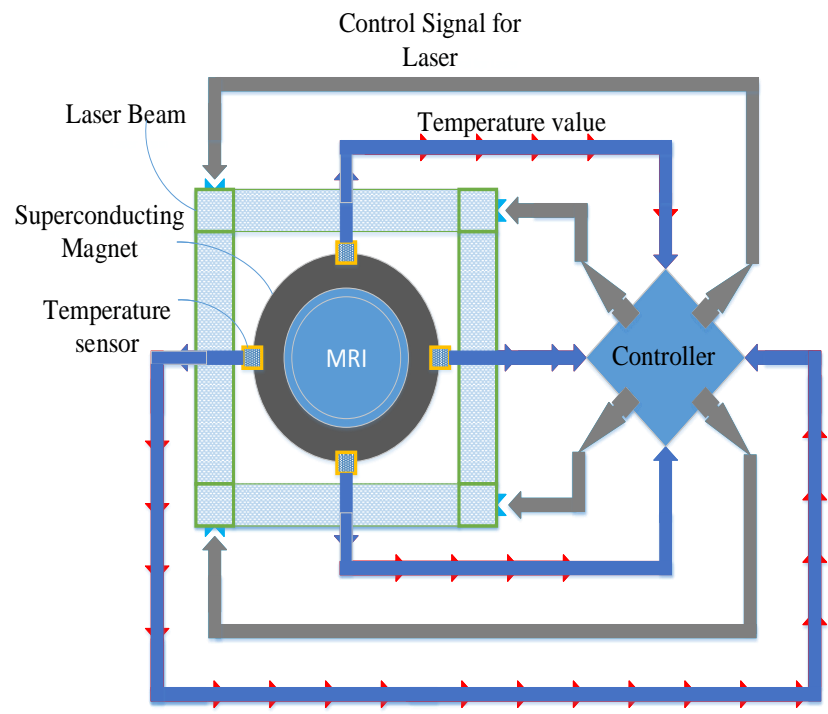

Fig. 2 Block diagram of LCS for MRI scanner

In this proposed system four temperature sensors are fixed on the four sides of the superconducting magnet. It can predict the temperature level at the superconducting magnet, and transmit it to the controller. So the controller has to be designed for making the cooling effective. And we have to place our model in controller so that it can provide the corresponding wavelength of laser for the predicted temperature. Here we were using Semiconductor Ring Laser (SRL).

\subsection{Semiconductor Ring Laser (SRL)}

Semiconductor Ring Lasers are miniature ring laser devices with potential applications in optoelectronics, photonics and all optical circuits. SRLs are literally ring shaped optical waveguides with a lasing medium. The structure of the tunable SRL is shown in Fig. 3.

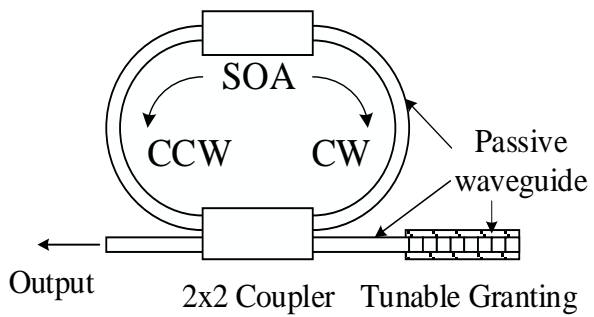

Fig. 3 Structure of tunable SRL

SRL has the ability to trap light in a ring, and recirculate it continuously as long as they remain powered.

\subsection{Modeling of LCS with Normal Controller}

The Fig. 4 shows the modeling of LCS with normal controller.

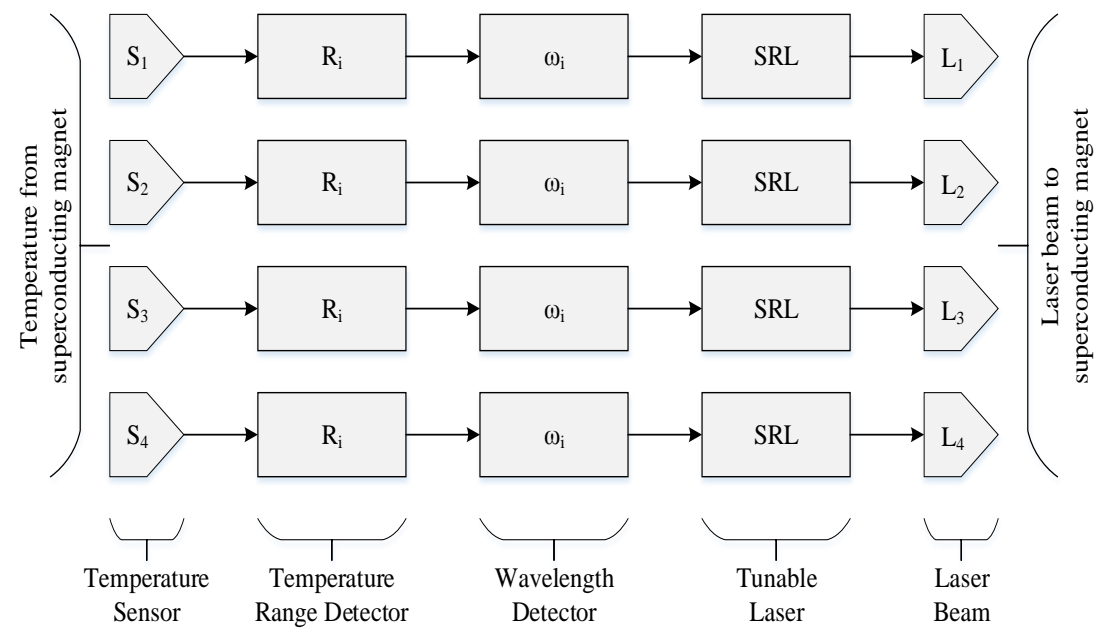

Fig. 4 Block diagram of LCS for MRI scanner with normal controller

Here the temperature sensor is a device that provides for temperature measurement through an electrical signal. The detected temperature of superconducting magnet from the temperature sensor is applied to the temperature range detector. The founded temperature range is given to the wavelength detector. The wavelength is supplied to the corresponding lasers. Finally they generate the laser beam which cools the superconductive magnet. The temperature detectors and wavelength detectors are collectively called as controller. The process diagram of Normal Controller for LCS is shown Fig. 5.

In this paper we ranged the temperature from 0kelvin to 20 Kelvin in five ranges (R1,R2,R3,R4,R5). So the detected temperature $\mathrm{T}$ is ranged under any one of the given five ranges. The particular range of the detected temperature is finding out based on the equation given below.

$$
R=\left\{\begin{array}{l}
\mathrm{R} 1, \text { if } \mathrm{T}=0 \mathrm{~K} \\
\mathrm{R} 2 \text {, if } 1 \mathrm{~K} \leq \mathrm{T}<5 \mathrm{~K} \\
\mathrm{R} 3, \text { if } 5 \mathrm{~K} \leq \mathrm{T}<10 \mathrm{~K} \\
\mathrm{R} 4, \text { if } 10 \mathrm{~K} \leq \mathrm{T}<15 \mathrm{~K} \\
\mathrm{R} 5, \text { if } 15 \mathrm{~K} \leq \mathrm{T}<20 \mathrm{~K}
\end{array}\right.
$$

The wavelength is detected based on the given equation

$$
\omega=\left\{\begin{array}{l}
\text { Onm for R1 } \\
478 \mathrm{~nm} \text { for R2 } \\
496 \mathrm{~nm} \text { for R3 } \\
514 \mathrm{~nm} \text { for R4 } \\
532 \mathrm{~nm} \text { for R5 }
\end{array}\right.
$$


The laser beam with corresponding wavelength is applied to the superconducting magnet, so the temperature is reduced and the new temperature level is sensed by sensor and the process is repeated till the MRI scanner is under operation. The updated temperature is given as TNEW $=\mathrm{T}-\mathrm{C}$, where TNEW is the new temperature after applying cooling, $\mathrm{T}$ is the current temperature and $\mathrm{C}$ is the corresponding cooling obtained by the application of laser with wavelength $\omega$.

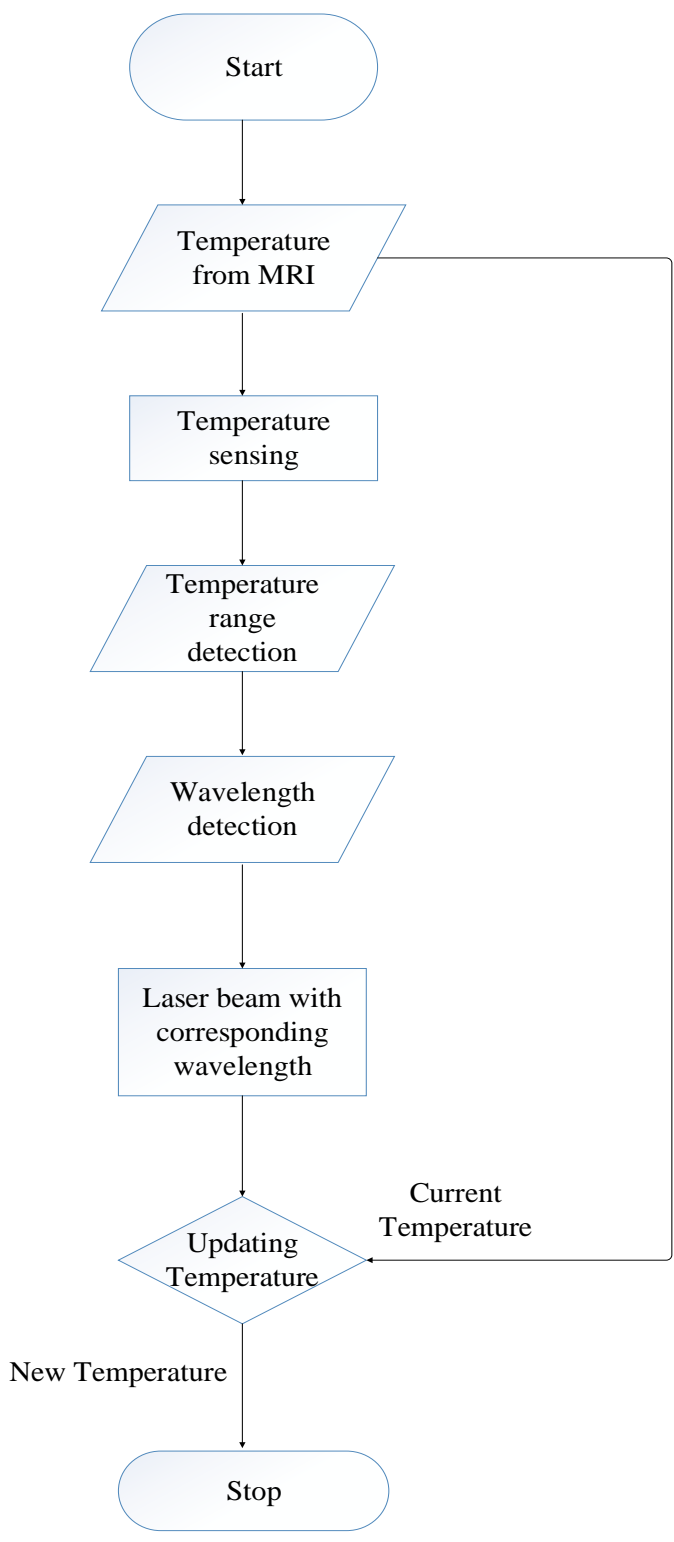

Fig. 5 Flow chart of normal controller for LCS

\section{RESULT AND DISCUSSION}

The proposed work is analyzed by three metrics namely Sensitivity, Specificity and Accuracy. The performance measure of normal controller is shown in Fig. 6.

\section{Performance with Normal controller}

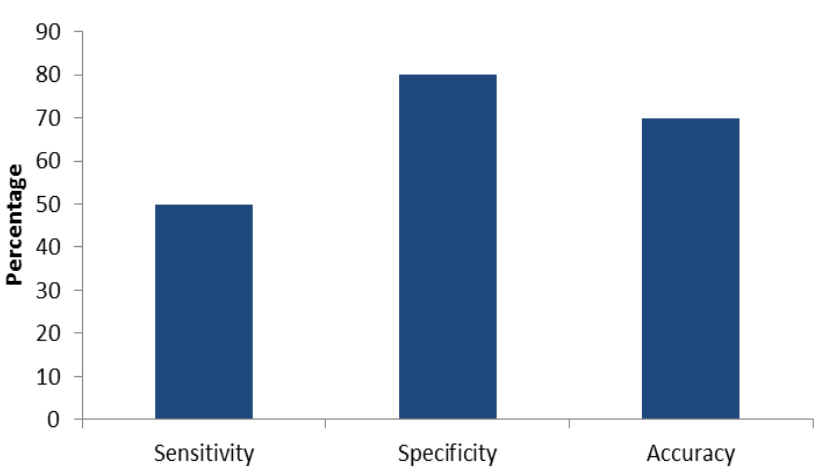

Fig. 6 Performance measure of LCS with normal controller

The Fig. 7 shows that the sensitivity of normal controller is $50 \%$, specificity of LCS with normal controller is $80 \%$ and Accuracy is $70 \%$. And the graph shown below gives the relation between temperature and time which results temperature reduction by normal controller.

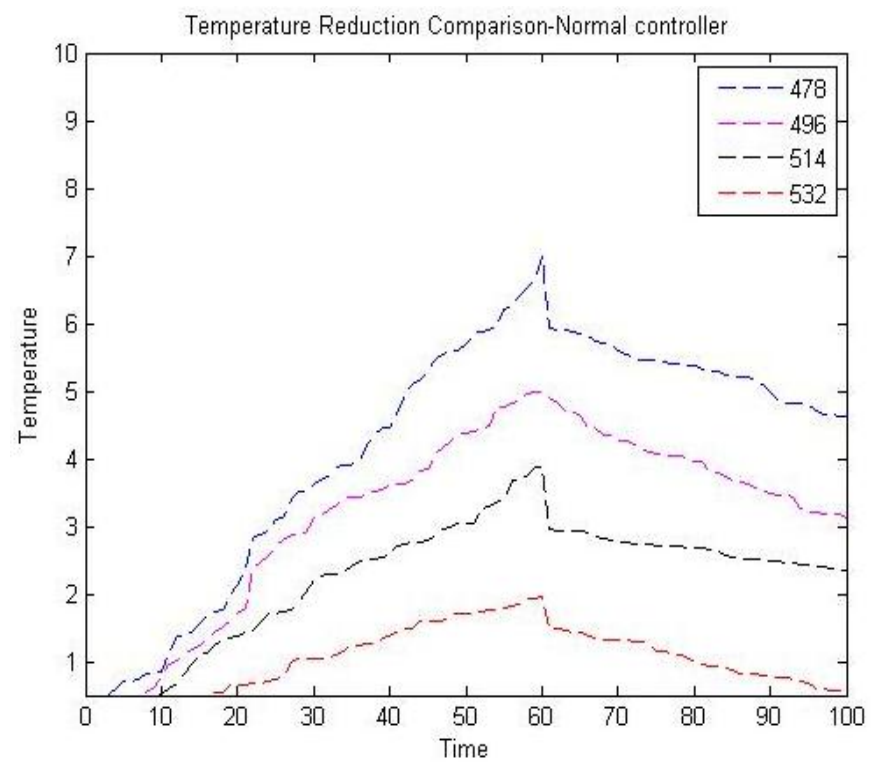

Fig. 7 Temperature reduction comparison at different wavelength by normal controller

\section{CONCLUSSION}

MRI is a medical imaging technique used in radiology to investigate the anatomy and physiology of the body in both health and disease. The most important and expensive part of MRI scanner is the superconducting magnet. During the operation of MRI scanner, large amount of heat is generated because of the handling of large magnetic field. Hence an efficient cooling system is for cooling the superconducting magnet. The conventional cooling system uses around 1,700 liters of liquid helium in a typical MRI scanner. Helium is the second most abundant material in the universe. And the handling of liquid helium is also very difficult. The proposed system for MRI scanner using laser provides optimal cooling as LCS cool the system to around 10kelvin and reduce the cost and handling complexity. 


\section{REFERENCES}

[1] Thomas C. Cosmus and Michael Parizh, "Advances in Whole-Body MRI Magnets", IEEE Transactions on Applied Superconductivity, Vol. 21, No. 3, pp. 2104-2109, 2011.

[2] Walter Koechner, "Solid-state laser engineering", Springer Book, Vol. 1, 2006.

[3] Todd A. Gould, "How MRI works", How Stuff Works. Inc., Vol. 10, 2008.

[4] William V. Hassenzahl, Drew W. Hazelton, Brian K. Johnson, Peter Komarek, Mathias Noe and Chandra T. Reis, "Electric power applications of superconductivity", Proceedings of the IEEE, Vol. 92, No. 10, pp. 1655-1674, 2004

[5] Michael Curzon Green, "Radiotherapy machine including magnetic resonance imaging system", U.S. Patent 6,198,957, issued March 6, 2001.

[6] Olivia Solon, "Cooling MRI magnets without a continuous supply of scarce helium", Technology, August 2013, from http://www.wired.co.uk/news/archive/2013-08/12/mrimagnet-cooling

[7] Roshini T V and Dr. N. Muruganand. "Electronic Cooling By LASER". IJCA Proceedings on International Conference on VLSI, Communications and Instrumentation (ICVCI) (8):1-3, 2011.

[8] William Thomas Silfvast, "Laser fundamentals", New York: Cambridge University Press, Vol. 459, 1996.

[9] TheodorW.Hänsch and Arthur L. Schawlow, "Cooling of gases by laser radiation", Optics Communications, Vol. 13 , No. 1 , pp. $68-69,1975$.

[10] C. Cohen-Tannoudji and William D. Phillips, "New mechanisms for laser cooling", Physics Today, Vol. 43, No. 10, pp. 33-40, 1990.

[11] Informed Decision Making Trumps Informed Consent for Medical Imaging with Ionizing Radiation James A. Brink, MD, Marilyn J. Goske, MD, and John A. Patti, MD January 2012 Volume 262, Issue 1
[12] Moderate dose rate ionizing radiation increases longevity (C) The British Institute of Radiology J R Cameron, $\mathrm{PhD}$, Departments of Medical Physics, Radiology and Physics, University of Wisconsin, Madison WI, USA DOI: http://dx.doi.org/10.1259/bjr/62063624 Published Online: January 28, 2014.

[13] R. Damadian, M. Goldsmith and L. Minkoff, "NMR in cancer: XVI Fonar image of the live human body", Physiological Chemistry and Physics, Vol. 9, pp. 97-100, 1977.

[14] https://nationalmaglab.org/education/magnetacademy/learn-the-basics/stories/mri-a-guided-tour

[15] Dalibard, J. and Cohen-Tannoudji, C., 1989. "Laser cooling below the Doppler limit by polarization gradients: simple theoretical models". JOSA B, Vol 6, No 11,pp.2023-2045.

[16] Laser cooling and trapping of neutral atoms Nobel Lecture by William D. Phillips, Dec 8, 1997, doi: 10.1103/RevModPhys.70.721.

[17] Massachusetts Institute of Technology (2007,April 8). Laser-cooling Brings Large Object Near Absolute Zero. ScienceDaily. Retrieved January 14, 2011.

\section{AUTHOR PROFILE}

Roshini T V , obtained her B-Tech Govt. College of Engineering, Kannur, Kerala, India, M-Tech from National Institute of Technology, Calicut, India. She is currently pursuing her $\mathrm{PhD}$ in Laser cooling system of MRI scanner. And also employed as Associate Professor, Vimal Jyothi Engineering College Chemperi, Kannur, Kerala, India. Her special field of interest includes Laser Cooling and Artificial Neural Networks

Dr. Kamalraj Subramaniam, obtained his BE from Karpagam College of Engineering, Anna university Chennai, India, ME from Anna university, Chennai, India and $\mathrm{PhD}$ from Universiti Malaysia Perlis, Malaysia. He is currently employed as Associate Professor, Karpagam Academy of Higher Education. His research interest includes Bio-medical signal processing \& VLSI Design. 